\title{
The impending conversion factor crisis and neurointerventional practice
}

\author{
Theresa H Nguyen 다, ${ }^{1}$ Joshua A Hirsch 이, ${ }^{2}$ Melissa M Chen, ${ }^{3}$ \\ Lauren Golding, ${ }^{4}$ Thabele M Leslie-Mazwi ${ }^{10},{ }^{5,6} \mathrm{Greg} \mathrm{N}$ Nicola, ${ }^{7}$ \\ Clemens M Schirmer (1) , ${ }^{8,9}$ James M Milburn ${ }^{1}$
}

\section{INTRODUCTION}

On August 3, 2020, the Centers for Medicare and Medicaid Services (CMS) released the 2021 Medicare Physician Fee Schedule (MPFS) Proposed Rule. The MPFS is used by CMS to calculate physician reimbursement, generating relative value unit (RVU) numbers that are adjusted for geography. The 2021 edition entailed reducing the RVU conversion factor (CF) from \$36.09 to $\$ 32.26$, (a $-10.61 \%$ change). Due to budget neutrality requirements, the reduction is mainly due to increased expenditures for evaluation and management (E/M) services. Using neurosurgery and interventional radiology (IR) as predicates for NeuroInterventional (NI) practice, were the 2021 MPFS Proposed Rule to be implemented, we would predict reimbursement decreases of approximately 7\%-9\% from CMS. To understand why the 2021 CMS proposed rule results in this substantial decrease for neurointerventionalists, we must recognize how physicians are compensated by CMS and the impetuses for change in payment for office/outpatient services that have been brewing over the past few years.

\section{HOW CMS DETERMINES PHYSICIAN PAYMENTS}

In brief, payment for physician services by the CMS is correlated to the number

\footnotetext{
${ }^{1}$ Department of Radiology, Ochsner Medical System,

New Orleans, Louisiana, USA

${ }^{2}$ Department of Neurolnterventional Radiology,

Massachusetts General Hospital, Boston,

Massachusetts, USA

${ }^{3}$ Department of Neuroradiology, University of Texas MD Anderson Cancer Center, Houston, Texas, USA

${ }^{4}$ Triad Radiology Associates PLLC, Winston-Salem, North Carolina, USA

${ }^{5}$ Department of Neurosurgery, Massachusetts General

Hospital, Boston, Massachusetts, USA

${ }^{6}$ Department of Neurology, Massachusetts General

Hospital, Boston, Massachusetts, USA

${ }^{7}$ Hackensack Radiology Group, Hackensack, New Jersey, USA

${ }^{8}$ Department of Neurosurgery and Neuroscience Institute, Geisinger, Wilkes-Barre, Pennsylvania, USA

${ }^{9}$ Research Institute of Neurointervention, Paracelsus Medical University, Salzburg, Austria

Correspondence to Dr Theresa H Nguyen, Department of Radiology, Ochsner Clinic Foundation, New Orleans, LA 70121, USA; Theresa.nguyen@ ochsner.org
}

of RVUs allocated for that service, which takes into account physician work (professional component), practice expense (technical component), and professional liability insurance. The RVU is multiplied by a conversion factor, set by CMS to maintain budget neutrality, resulting in the eventual dollar amount paid for a service. ${ }^{1}$ To consistently describe medical services, a unique and billable Current Procedural Terminology (CPT) code are assigned. Codes are allocated RVUs by CMS based on input from the AMA Relative Value Scale Update Committee (RUC). ${ }^{2}$ CMS updates and publishes payment for physician services annually in the Medicare Physician Fee Schedule (MPFS). New codes are periodically added to the MPFS, and the number of RVUs assigned can also be adjusted to more accurately reflect the value of that service. ${ }^{45}$ The MPFS must be budget-neutral, so increasing payments for certain services invariably decreases those for others. In the 2021 Proposed Rule, the nearly $11 \%$ conversion factor reduction is mostly due to increased expenditures for office/outpatient $\mathrm{E} / \mathrm{M}$ services while keeping budget neutrality.

\section{HOW THE PAST THREE YEARS HAVE LED US TO THE 2021 CONVERSION FACTOR}

The 2021 MPFS is primarily shaped by an effort to restructure $\mathrm{E} / \mathrm{M}$ codes over the past 3 years to simplify billing and documentation requirements. Evaluation and Management codes, as the name suggests, are a category of CPT codes that describe non-procedural services billed for by physicians and other providers. Sometimes this has been grouped as "cognitive work." Payment for these services is based on the E/M visit's complexity level, which ranges from 1 (the lowest value) to 5 (the highest). The complexity level is represented by a CPT code (99201-99205 for new outpatient visits; 99211-99215 for established outpatient visits). To bill for a specific complexity level, a provider must adhere to documentation requirements for the patient's history, physical examination, medical decision making, and time spent on the visit. In 2017, CMS launched the "Patients over Paperwork" initiative to reduce regulatory burdens to providers. This led to the 2019 MPFS Final Rule eliminating specific documentation requirements for $\mathrm{E} / \mathrm{M}$ services and consolidated levels 2-4 E/M services into a single blended rate. New add-on codes were also created to reflect complex visits in order to offset payment decreases expected with the consolidation of levels 2-4 E/M services for some specialties caring for more involved patients. ${ }^{6}$

The 2019 MPFS Final Rule was held from implementation because of widespread concerns that the consolidated payment levels unfairly disadvantaged certain specialties who care for higher complexity patients. This concern prompted the AMA to develop alternative solutions to the 2019 MPFS Final Rule. ${ }^{7}$ Notable recommendations from the AMA included: preserving five levels of $\mathrm{E} / \mathrm{M}$ services for established patients; consolidating levels $1 \& 2 \mathrm{E} / \mathrm{M}$ services for new patients; and creating a new add-on code (99 XXX) for extended visits. These code changes were revalued at the RUC meeting in April 2019, which agreed with the AMA and allocated higher RVUs to E/M codes, with some work RVU increases of up to $46 \%{ }^{6}$

Most of the AMA and RUC proposals were accepted by CMS and put forth in the 2020 MPFS Proposed Rule. Under the new coding framework, CMS maintains the five different service levels, which would now be determined by either medical decision making or time spent on the visit, removing the history and examination component. The revised $\mathrm{E} / \mathrm{M}$ codes are accompanied by higher work RVUs, which increases payment for these services. $\mathrm{E} / \mathrm{M}$ visits are estimated to comprise approximately $40 \%$ of allowed charges for MPFS services, and office/outpatient $\mathrm{E} / \mathrm{M}$ visits, in particular, comprise approximately $20 \%$ of allowed charges. ${ }^{1}$ Since E/M services make up such a large percentage of Medicare spending, changes to their valuations significantly impact the MPFS budget distribution. Because of the magnitude of these changes in the 2020 Proposed Rule, their effective date was scheduled for January 1, 2021, to allow the medical industry time to prepare for the transition. ${ }^{8}$

\section{PROPOSED RULE AND THE CONVERSION FACTOR}

One year later, the 2021 Proposed Rule further increased $\mathrm{E} / \mathrm{M}$ expenditures by revaluing services CMS deemed analogous to outpatient/office visits. These include transitional care management, 
certain end-stage renal disease services, annual wellness visits, preventative physical examinations, emergency department visits, cognitive impairment assessments, care planning, therapy evaluations, psychiatric and psychotherapy services, and maternity services. CMS states these services either had E/M visits explicitly built into their valuations or were valued using a direct crosswalk to the RVUs assigned to an office/outpatient visit. Thus, per CMS, their values should be adjusted commensurate with changes in value for office/outpatient $\mathrm{E} / \mathrm{M}$ visits. ${ }^{1}$ In contrast, CMS did not apply these increased RVU valuations to the E/M components of global surgery codes (with the exception of maternity surgical packages) citing "we (CMS) continue to collect and analyze the data on the number and level of office/outpatient $\mathrm{E} / \mathrm{M}$ visits that are actually being performed as part of these services."

Additionally, new codes were created for $\mathrm{E} / \mathrm{M}$ services, aiming to better describe the cognitive work related to care management and coordination, such as add-on code 99 XXX which describes prolonged office visits and can be billed when the maximum time for a level 5 office/ outpatient visits is exceeded by at least 15 min. CMS included another add-on code, GPC1X, which designates visit complexity inherent to coordinating and maintaining continuity of care of complex patients. GPC1X was originally proposed alongside consolidation of $\mathrm{E} / \mathrm{M}$ levels 2-4 in order to offset payment decreases for providers taking care of complex patients. ${ }^{6}$ In the 2021 Proposed Rule, CMS asserts the revised five levels of $\mathrm{E} / \mathrm{M}$ codes do not adequately capture the work associated with individual visits and offer add-on coding for complex encounters. ${ }^{1}$ However, there is no utilization data for the revised $\mathrm{E} / \mathrm{M}$ codes or the new add-on codes and the justification for adding this remains purely conceptual with ambiguous criteria, making it susceptible to billing with any visit. This could result in overutilization and has the downstream effect of further impact on the conversion factor in future years.
To maintain budget neutrality, the substantial increase in RVUs for $\mathrm{E} / \mathrm{M}$ care is offset by redistributing RVUs from other services and decreasing the conversion factor a historic $10.6 \%$ to $\$ 32.26$. The CF change reduces the monetary value of all RVUs, but payments for office/outpatient $\mathrm{E} / \mathrm{M}$ services will increase because of their higher allotted RVUs. The impact of the change to the conversion factor (based on historical claims data) that CMS estimates will occur to base specialties is noted in table 1 . NI surgery is a field comprised of three base specialties which are included in table 1 . In terms of the conversion factor, we believe that interventional radiology and neurosurgery are reasonable proxies for neuroendovascular practice. CMS estimates payment decreases of $9 \%$ and $7 \%$, respectively for IR and neurosurgery. Thus, neurointerventionalists as a discipline will likely experience payment decreases in that range. Specialties providing fewer $\mathrm{E} / \mathrm{M}$ services will experience even deeper cuts.

An additional consideration in attempts to predict the impact of these changes is the incorporation of telehealth services. The ongoing COVID-19 pandemic dramatically accelerated telehealth service utilization including among neurointerventionalists. ${ }^{9}$ As a result of their popularity with beneficiaries and providers, CMS proposed to permanently add eight new codes to the Medicare telehealth services list for 2021. During the public health emergency, CMS and many private payers have agreed to reimburse telehealth office visits at parity with in-person office/outpatient-based E/M services. With improvements in technology, it seems plausible that telemedicine utilization will grow even after the pandemic ends. The effect on reimbursement for neurointerventionalists may relate to the inflation of these services in contrast to others, something that is difficult to determine at this point.

This expansion of $\mathrm{E} / \mathrm{M}$ services with telehealth, new add-on codes and revaluation of similar services will likely create further challenges to the conversion factor in future years. Moreover, the impact on practice may reverberate: although the $\mathrm{CF}$ pertains to Medicare payments, private insurers often base their rates on those set by Medicare. Thus, the 2021 Proposed Rule will likely have ripple effects throughout the private payer market, and neurointerventionalists may see decreased negotiated rates with commercial insurers as well.

Medicare payments have not kept pace with inflation: between 1992 (the start of the resource-based RVU system) and 2016, Medicare payments have decreased by 53\%. Furthermore, Medicare has decreased physicians' payments by almost $25 \%$ just between 2001 and 2018 relative to inflation. ${ }^{10}$ The proposed CF $10.61 \%$ decrease in 2021 to $\$ 32.26$ is an unprecedented reduction, bringing the CF close to 1992 when it was $\$ 31.00$. Adjusted for inflation, the 2021 proposed amount would be even lower. The \$31.00 in 1992 is equivalent in purchasing power to approximately $\$ 57.43$ in 2020 , a difference of $\$ 26.43$ over 28 years. Reducing the CF back to its 1992 figure is an unrealistic solution for addressing contemporary healthcare issues, given the substantial loss of reimbursement already occurring due to the COVID-19 pandemic. We believe the most appropriate course of action would be to temporarily suspend the budget neutrality requirement so that access to quality NI care is preserved.

The COVID-19 pandemic highlighted the shortcomings in healthcare delivery in the United States of primary and specialty care services - including acute stroke management performed by neurointerventionalists. ${ }^{11}$ The anticipated significant cuts in Medicare payments to neurointerventionalists jeopardize patient access to lifesaving therapies. In response to the 2021 Proposed Rule, the SNIS has joined a coalition of professional societies urging Congress to prevent the proposed Medicare physician payment cuts scheduled to take effect on January 1, 2021. Absent suspension of the budget neutrality requirement, we fear the access challenges that might accompany such a draconian change.

Table 1 Estimated specialty impact of CMS 2021 proposed rules (adapted from table 90) ${ }^{1}$

\begin{tabular}{llllll}
\hline Specialty & $\begin{array}{l}\text { Allowed charges (US\$, } \\
\text { million) }\end{array}$ & Impact of work RVU changes & Impact of PE RVU changes & Impact of MP RVU changes & Combined impact \\
\hline Diagnostic radiology & 5253 & $-6 \%$ & $-5 \%$ & $0 \%$ & $-11 \%$ \\
Interventional radiology & 497 & $-3 \%$ & $-5 \%$ & $0 \%$ & $-9 \%$ \\
Neurology & 1513 & $3 \%$ & $2 \%$ & $0 \%$ & $-1 \%$ \\
\hline Neurosurgery & 806 & $-4 \%$ & $-2 \%$ & $-1 \%$ & $-7 \%$ \\
\hline
\end{tabular}


Twitter Theresa H Nguyen @TheNguyenMD, Joshua A Hirsch @JoshuaAHirsch, Melissa M Chen @ MelissaChenMD and James M Milburn @docroc99

Contributors $\mathrm{THN}, \mathrm{JAH}$, and JMM drafted the initial manuscript. All authors provided editorial suggestions and approved the final version.

Funding The authors have not declared a specific grant for this research from any funding agency in the public, commercial, or not-for-profit sectors.

Competing interests JAH serves as Chair of the Society of Neurolnterventional Surgery Health Policy Committee. CMS serves as an adviser to the AMA RVBS Update Committee on behalf of the Congress of Neurological Surgeons.

Patient consent for publication Not required.

Provenance and peer review Not commissioned; internally peer reviewed.

Data availability statement All data relevant to the study are included in the article. Data from Table 1 of this article is derived from the CMS 2021 Proposed Rule-Table 90 . This data is publicly available from: https://www.federalregister.gov/documents/2020/08/ 17/2020-17127/medicare-program-cy-2021-paymentpolicies-under-the-physician-fee-schedule-and-otherchanges-to-part.

(C) Author(s) (or their employer(s)) 2021. No commercial re-use. See rights and permissions. Published by BMJ.

\section{Check for updates}

To cite Nguyen TH, Hirsch JA, Chen MM, et al. J Neurolntervent Surg 2021;13:301-303.

Accepted 30 October 2020

Published Online First 30 November 2020
J Neurolntervent Surg 2021;13:301-303. doi:10.1136/neurintsurg-2020-017005

ORCID iDs

Theresa H Nguyen http://orcid.org/0000-0003-05002577

Joshua A Hirsch http://orcid.org/0000-0002-9594-8798 Thabele M Leslie-Mazwi http://orcid.org/0000-00024191-2466

Clemens M Schirmer http://orcid.org/0000-0003-17438781

\section{REFERENCES}

1 Center for Medicare \& Medicaid Services. Proposed Rule: Medicare Program; CY 2021 Payment Policies Under the Physician Fee Schedule and Other Changes to Part B Payment Policies; Medicare Shared Savings Program Requirements; Medicaid Promoting Interoperability Program Requirements for Eligible Professionals; Quality Payment Program; Coverage of Opioid Use Disorder Services Furnished by Opioid Treatment Programs; Medicare Enrollment of Opioid Treatment Programs; Electronic Prescribing for Controlled Substances for a Covered Part D Drug Under a Prescription Drug Plan or an MA-PD Plan; Payment for Office/Outpatient Evaluation and Management Services; Hospital IQR Program; Establish New Code Categories; and Medicare Diabetes Prevention Program (MDPP) Expanded Mode Emergency Policy CMS-1734-P. [Internet], 2020. Available: https://www.govinfo.gov/content/pkg/FR2020-08-17/pdf/2020-17127.pdf [Accessed 18 Aug 2020].

2 Hirsch JA, Leslie-Mazwi TM, Nicola GN, et al. Current procedural terminology; a primer. J Neurointerv Surg 2015;7:309-12

3 Hirsch JA, Silva E, Nicola GN, et al. The RUC: a primer for neurointerventionalists. J Neurointerv Surg 2014:6:61-4.
4 Hirsch JA, Donovan WD, Leslie-Mazwi TM, et al. Component coding and the neurointerventionalist: a tale with an end. J Neurointerv Surg 2013;5:615-9.

5 Donovan WD, Leslie-Mazwi TM, Silva E, et al. Diagnostic carotid and cerebral angiography: a historical summary of the evolving changes in coding and reimbursement in a complex procedure family. $J$ Neurointerv Surg 2014;6:712-7.

6 Wang KY, Hirsch JA, Nicola GN, et al. Implications of the revisions and revaluation of office/outpatient evaluation and management codes for neuroradiology reimbursement. AJNR Am I Neuroradiol 2020;41:1160-4.

7 American Medical Association. CPT evaluation and management [Internet]. Available: https://www.amaassn.org/practice-management/cpt/cpt-evaluationand-management [Accessed 11 Oct 2020].

8 Center for Medicare \& Medicaid Services. Revisions to Payment Policies under the Medicare Physician Fee Schedule, Quality Payment Program and Other Revisions to Part B for CY 2020. CMS-1715-F. [Internet], 2019. Available: https://www.federalregister. gov/documents/2019/11/15/2019-24086/medicareprogram-cy-2020-revisions-to-payment-policies-underthe-physician-fee-schedule-and-other [Accessed 16 Oct 2020].

9 Rai AT, Frei D. A rationale and framework for seeking remote electronic or phone consent approval in endovascular stroke trials - special relevance in the COVID-19 environment and beyond. I Neurointerv Surg 2020;12:654-7.

10 Golding LP, Rosenkrantz AB, Nicola GN, et al. How radiology maintains relative value units but could lose big in reimbursement: the power of the conversion factor. J Am Coll Radiol 2020:17:542-5.

11 Schirmer CM, Ringer AJ, Arthur AS, et al. Delayed presentation of acute ischemic strokes during the COVID-19 crisis. J Neurointerv Surg 2020;12:639-42. 